\title{
Maturity work: Towards a copper-azophloxine inorganic complex for the selective naked-eye detection of pyrophosphate in water
}

\author{
Aria Bieria ${ }^{a}$, Thibaud Rossel $\left.\right|^{a, b}, 1,2$ \\ a Gymnase français de Bienne, \\ Pré Jean-Meunier 1, Moutier, 2740, Switzerland \\ b Institute of Chemistry, University of Neuchâtel, \\ Avenue de Bellevaux 51, Neuchâtel, 2000, Switzerland
}

\section{Biography of Aria Bieri}

Aria Bieri (Figure 1) is a high school student of the Gymnase français de Bienne. She is part of the école de maturité spécialisée section where she learns chemistry. For her maturity work, she is interested in building new optochemical compounds for sensing. The title of her work is: "Copper Complexes for the Visual Detection of Pyrophosphate". Aria is a very enthusiastic and curious student. Outside of her school hours, she likes doing yoga, dancing and cooking for her family and friends.

\section{Abstract}

Indicator displacement assay is a well-known methodology reported for the detection of analytes. It is very easy to perform, especially for high school students. Since the methodology is simple, wide libraries can be generated with such a strategy. Potentially, it could provide interesting chemosensors that were never reported before in the literature on the naked-eye detection of a specific analyte of interest. Here for a maturity work, we investigated the possibility of detecting pyrophosphate with an indicator displacement assay. In the first instance, we combined copper with azophloxine to form an inorganic complex in water. The chemosensor which is not reported in the literature is able to detect pyrophosphate at micromolar concentration with a concomitant colour change. In the second instance, we screened a collection of anions to investigate the selectivity of our chemosensor. Our results showed that our sensor is very specific to pyrophosphate and doesn't suffer from interactions with phosphate or carbonate. Overall our results indicate that real research with socially relevant results can be conducted at high school by using smart methodologies and very modest resources.

\section{Introduction}

Indicator displacement assays have proven their utility in chemistry. [1] The methodology is widely used for various application such as, sensing [2,3], determination of the enantiomeric excess [2] or monitoring the cellular metabolism [4] for example. In this context, large combinatorial libraries with metal salts

\footnotetext{
${ }^{1}$ Corresponding author.

2E-mail: thibaud.rossel@unine.ch
} 


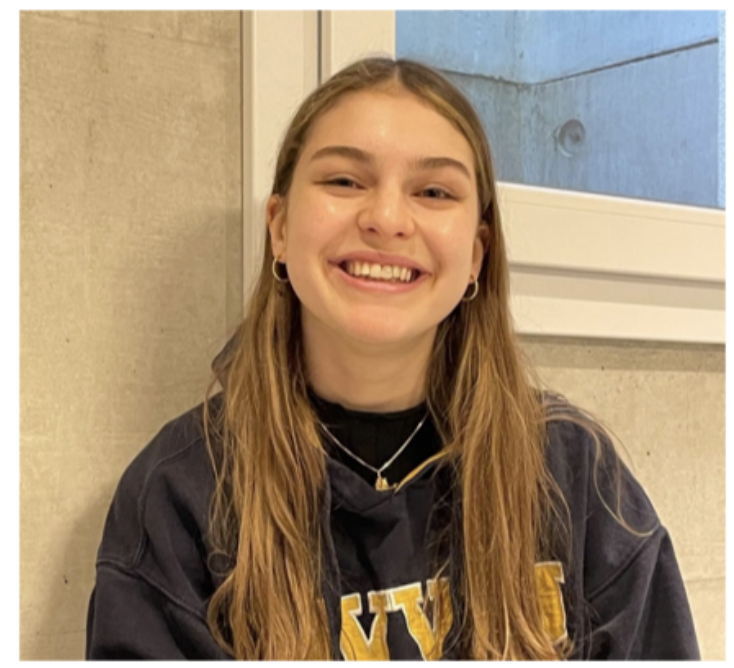

Figure 1. Aria Bieri

and indicators can be mixed and screened for a specific detection of analytes of interest. [5] Given the immense possibilities of the strategy, it is obvious that not all reactions are reported in the literature so far. However, their discovery could be useful for various and specific applications and contribute to the general knowledge in chemistry to stimulate the next generations. [5] For example, the simple detection of phosphate over pyrophosphate [6] is still a major challenge given the chemical properties of the first one e.g. large size of the anion, hydrophilicity, multiple protonation states, etc. [7] Areas of science with fundamental problems can be solved with this approach. We, for example, reported chemosensors for the naked-eye detection of phosphate or glyphosate in the past. [5, 7] Even if, indicator displacement assay is more of a procedure practiced for research at universities, it proved to be useful for research at high school too by developing a subcategory in the screening matrix that can be explored. Here, for example, we report a copper-based inorganic complex for the selective naked-eye detection of pyrophosphate in water. The complex has not been reported in the literature so far for sensing this anion. This work has been carried out by a high school student for her maturity work demonstrating that relevant research contributing to the general knowledge of chemistry can be performed at high school using very modest and commercial resources. [8] This work can easily be explored and expanded in the future by the next generations of students thus providing an interesting piece of research using transgenerationnal collaborations driving curiosity. [9] 


\section{Materials and Methods}

Dissolving azophloxine (AP, $250 \mu \mathrm{M})$ and copper sulfate $\left(\mathrm{CuSO}_{4}, 250 \mu \mathrm{M}\right)$ in 2-[4-(2-hydroxyethyl)-1- piperazinyl]ethanesulfonic acid (HEPES, $10 \mathrm{mM}, \mathrm{pH}=7.4)$ affords [Cu(AP)] $(250 \mu \mathrm{M})$, which is water-soluble and orange at working micromolar concentrations (see Figures 2 and 3 ).

Screening of various pHs from 5 to 8 allowed us to conclude that the best naked-eye discrimination between $\mathrm{AP}$ and $[\mathrm{Cu}(\mathrm{AP})]$ is obtained at $\mathrm{pH}=7.4$. We therefore selected $\mathrm{pH}=7.4$ for the continuation of our study.

Next, we hypothesized that the [Cu(AP)] complex could be used to assemble a receptor for phosphate derivatives, exploiting metal-ligand interactions for selective target recognition. To the best of our knowledge, this mononuclear complex has never been described before in the literature as a selective chemosensor for anions, especially phosphate derivatives.

In this case, the displacement of the receptor-bound AP by anions could be visually observed with the naked eye (see Figure 3). The competition assay developed here is illustrated schematically in Figures 2 and 3.

Additionally, we measured absorbance spectra of the formed complex in the presence and in the absence of pyrophosphate (see Figure 4 ). The AP ligand shows an absorbance peak at $505 \mathrm{~nm}$ [range of 400-1000 nm] whereas the formed complex displays an absorbance peak at $490 \mathrm{~nm}$ [range of 400-1000 $\mathrm{nm}$ ]. The addition of 5 eq. of pyrophosphate to [Cu(AP)] allows an indicator displacement assay thus recovering the initial absorbance spectra of AP.

In the next step, we screened a collection of anions against the copper-based inorganic complex [Cu(AP)]. Out of all the tested ions, only one was able to displace the AP ligand: pyrophosphate.

The addition of pyrophosphate anions to the aqueous solution of this purple ensemble (see Figure 5) resulted in the recovery of AP's spectroscopic properties (see Figures 3 and 4). The present sensor exhibits excellent selectivity towards pyrophosphate ions over other anions, including phosphate and carbonate ions (see Figure 5).

\section{Results and discussion}

From a chemical point of view, the results obtained overall suggest that the complex [Cu(AP)] can be used for the naked-eye detection of pyrophosphate. However, these preliminary data should be further investigated in many ways such as:

- Identifying the effects of the promiscuity of various groups of anions on the complex.

- Extending the screening matrix with phosphorylated molecules to investigate the selectivity of the complex.

- Trying to determine the detection limit abilities of this system

- Trying to determine the affinity constant of [Cu(AP)]for pyrophosphate and comparing it with chemosensors described in the litterature

- Trying to prove the mechanism of detection of this indicator displacement assay

- Analysing the effect of light and heat on this complex since the ligand is an azobenzene. Is a isomerisation possible?

These are some of the investigations that can be carried out by the next generations of students for their maturity work inspired by this research piece.

From a pedagogical point of view, this approach allowed the student to dive into chemistry research by exploring non-described compounds. She was able to use many fundamentals she learned during chemistry courses such as the basics of organic chemistry, inorganic chemistry, spectroscopy, stoichiometry, etc. She was also able to reproduce the results she obtained and therefore train and discuss the reproducibility of experiments. [10] This is a key point that should be addressed nowadays due to the expansion of scientific literature and reported scientific misconducts. $[11,12,13]$ As an extension, she was also able to design and discuss positive and negative controls that are fundamental for reliable science, which may be a key point in the education of young scientists. [14] She was also able to discuss the hypothesis of detection abilities of the complex against various anions and therefore train her mind to look the direction 


\section{Azophloxine (AP)}<smiles>CC(=O)Nc1cc(S(=O)(=O)[O-])cc2cc(S(=O)(=O)[O-])c(N=Nc3ccccc3)c(O)c12</smiles><smiles>CC(=O)Nc1cc(S(=O)(=O)[O-])cc2cc([N+](=O)[O-])c(N(C)C(=O)O)c(Nc3ccccc3)c12</smiles>

[Cu(AP)]

\section{Pyrophosphate}

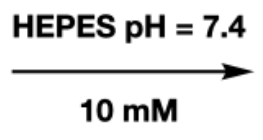

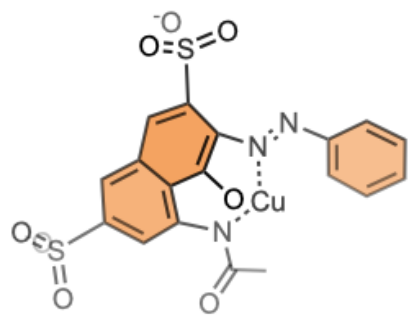

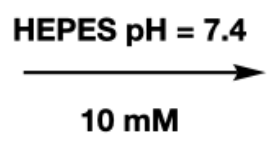

[Cu(AP)]

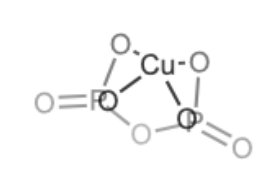

Figure 2. The proposed multi-step reaction mechanism presenting the indicator displacement assay based on

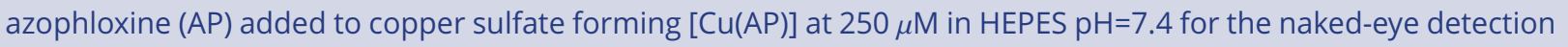
of pyrophosphate (10 mM HEPES pH=7.4).

of inorganic chemistry concepts. This allows the development of scientific critical thinking. $[15,16]$ Finally, as a woman in science, she was able to acquire confidence in herself, and this project allowed her to acquire the motivation to solve interesting scientific problems with social interests. [17] This is a key point since women are still underrepresented in sciences in general. [18, 19]

\section{Conclusion}

We have described a simple assay for the detection of pyrophosphate ions in an aqueous media. The assay is based on commercially available chemicals (copper sulfate and a colorimetric indicator) and allows detection of pyrophosphate at low micromolar concentration. The interference of common anions is remarkably low. Moreover, the assay is very easy to perform and requires no prior synthesis. The research project was realized as part of a maturity work realized by a high school student proving it's simplicity. The initial results suggest that interesting research with modest material can be performed with high school students. It also allows high school students to further the development of this project in various directions thus constructing interesting pieces of research that could be published ultimately. The project also allows the development of interesting and key pedagogical interests such as discussing reproducibility or positive and negative controls. Finally, it can also develop women's interest in the sciences 
A

B

C

D

E

$\mathbf{F}$
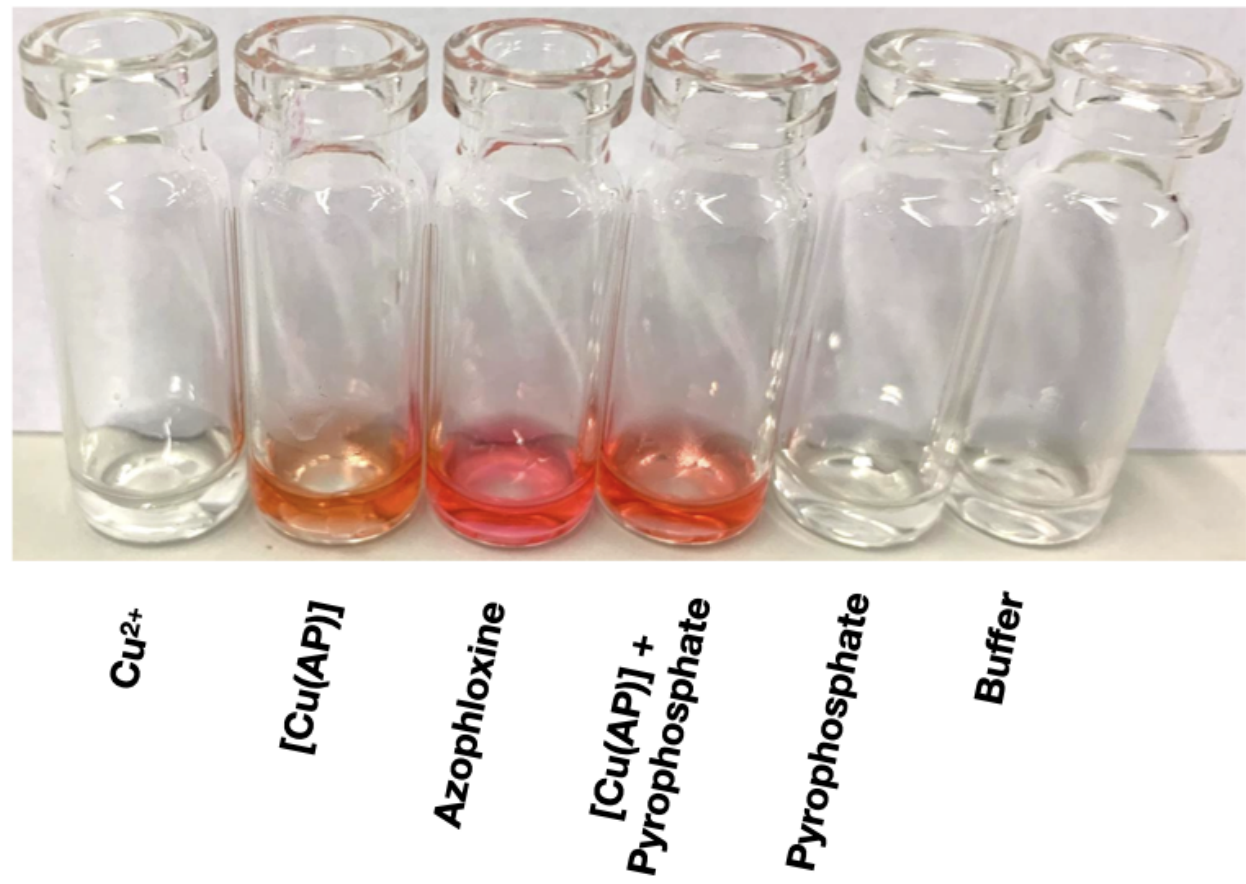

Figure 3. Addition of copper sulfate ( $\mathbf{A}, 250 \mu \mathrm{M}$, HEPES $\mathrm{pH}=7.4$ to azophloxine (B, AP, $250 \mu \mathrm{M}, \mathrm{HEPES} 10 \mathrm{mM}$ $\mathrm{pH}=7.4$ affords an inorganic complex. The addition of pyrophosphate to $\mathbf{B}$ (5 eq., HEPES $10 \mathrm{mM} \mathrm{pH=7.4)} \mathrm{allows} \mathrm{an}$ indicator displacement assay (D, $250 \mu \mathrm{M}$, HEPES $10 \mathrm{mM} \mathrm{pH=7.4).} \mathrm{C,} \mathrm{E} \mathrm{and} \mathbf{F}$ are negative controls.

\section{Acknowledgements}

The author would like to thank the Gymnase français de Bienne for lab equipment as well as support in this project. The author would to thank also the University of Neuchâtel and especially Prof. Dr. Bruno Therrien for discussions and support in the realisation of this project. Finally, all the students past and present that participated in this research program.

\section{References}

[1] Adam C Sedgwick, James T Brewster, Tianhong Wu, Xing Feng, Steven D Bull, Xuhong Qian, Jonathan L Sessler, Tony D James, Eric V Anslyn, and Xiaolong Sun. Indicator displacement assays (IDAs): the past, present and future. Chemical Society Reviews, 2021.

[2] Lei Zhu, Zhenlin Zhong, and Eric V Anslyn. Guidelines in implementing enantioselective indicatordisplacement assays for $\alpha$-hydroxycarboxylates and diols. Journal of the American Chemical Society, 127(12):4260-4269, 2005.

[3] Ning-Ning Yang, Li-Jiao Zhou, Peng Li, Qi Sui, and En-Qing Gao. Space-confined indicator displacement assay inside a metal-organic framework for fluorescence turn-on sensing. Chemical science, 10(11):3307-3314, 2019. 


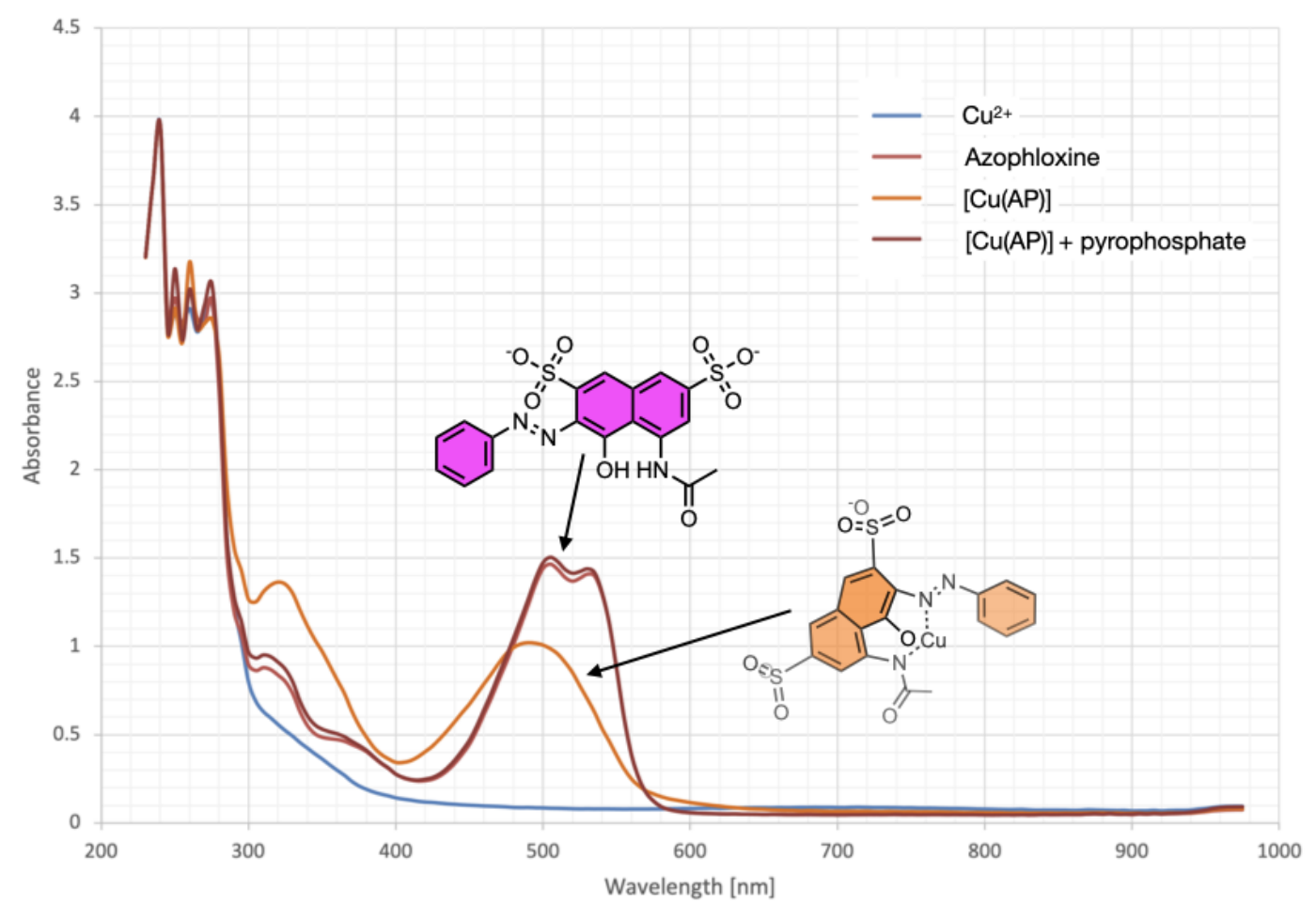

Figure 4. Absorbance spectra of [Cu(AP)] at $250 \mu \mathrm{M}$ formed in HEPES $\mathrm{pH}=7.4$ and in the presence of pyrophosphate (5 eq.).

[4] Amir Norouzy, Zahra Azizi, and Werner M Nau. Indicator displacement assays inside live cells. Angewandte Chemie International Edition, 54(3):792-795, 2015.

[5] Thibaud Rossel and Marc Creus. La Chimie en Couleurs: Socially Relevant and Original Research in Chemistry in High Schools Using Modest Resources. CHIMIA International Journal for Chemistry, 73(7-8):599-603, 2019.

[6] Prerna Yadav, Olivier Blacque, Andreas Roodt, and Felix Zelder. Induced fit activity-based sensing: a mechanistic study of pyrophosphate detection with a "flexible" Fe-salen complex. Inorganic chemistry frontiers, 8(19):4313-4323, 2021.

[7] Thibaud Rossel and Marc Creus. A biomimetic cerium-based biosensor for the direct visual detection of phosphate under physiological conditions. Chemical Communications, 55(99):14894-14897, 2019.

[8] George M. Whitesides. Using Simplicity, 042014.

[9] George M Whitesides. Curiosity and science. Angewandte Chemie International Edition, 57(16):41264129, 2018.

[10] Monya Baker. 1,500 scientists lift the lid on reproducibility. Nature News, 533(7604):452, 2016.

[11] Daniele Fanelli. Opinion: Is science really facing a reproducibility crisis, and do we need it to? Proceedings of the National Academy of Sciences, 115(11):2628-2631, 2018. 


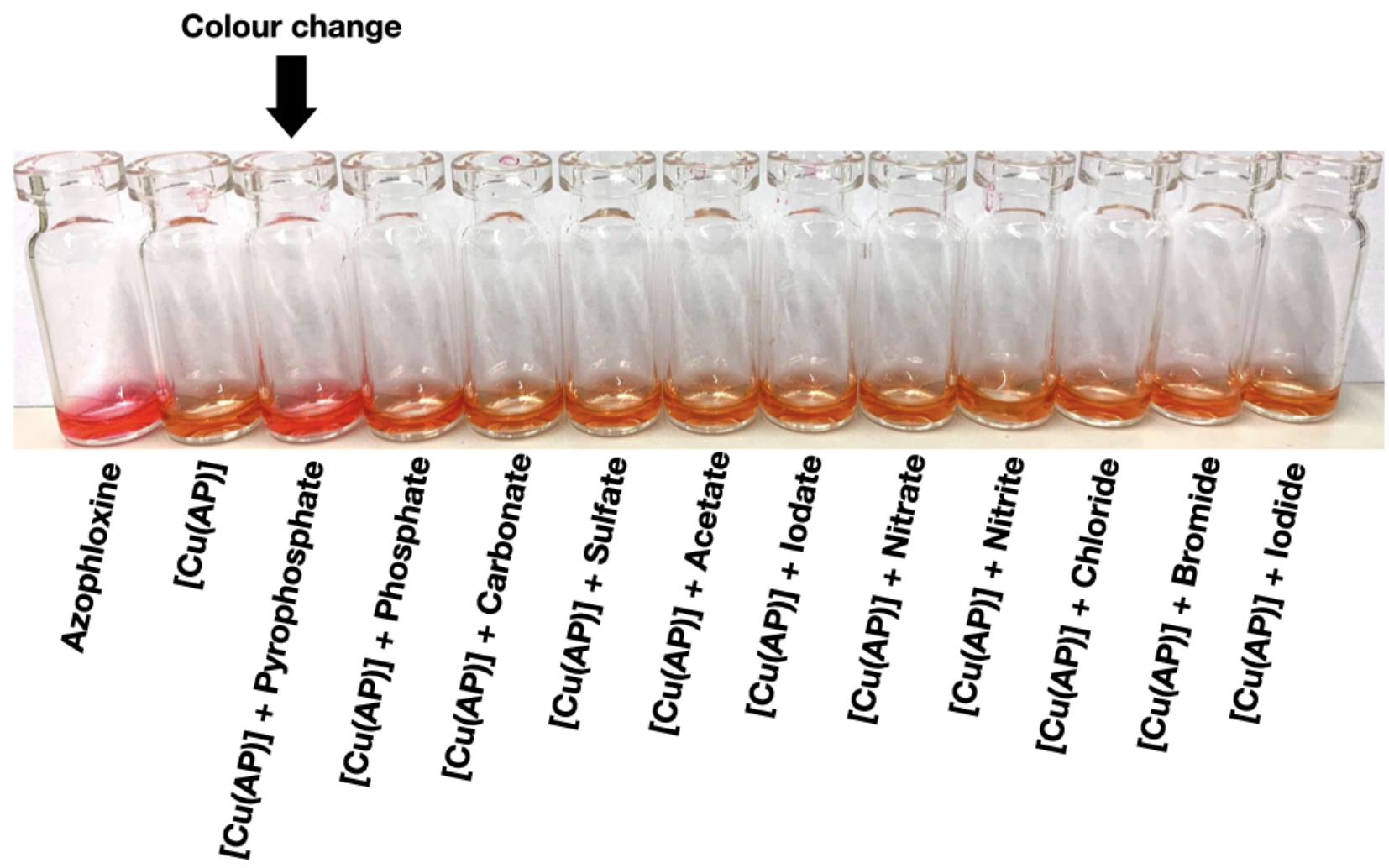

Figure 5. Screening of [Cu(AP)](250 $\mu \mathrm{M}$ in HEPES $10 \mathrm{mM}$ at $\mathrm{pH}=7.4$, aqueous solution) in the presence of various anions (1.25 mM, 5 equivalents).

[12] Jeremy Berg. Progress on reproducibility, 2018.

[13] Jay J Van Bavel, Peter Mende-Siedlecki, William J Brady, and Diego A Reinero. Contextual sensitivity in scientific reproducibility. Proceedings of the National Academy of Sciences, 113(23):6454-6459, 2016.

[14] John S Torday and František Baluška. Why control an experiment? From empiricism, via consciousness, toward Implicate Order. EMBO reports, 20(10):e49110, 2019.

[15] Susan A Seibert. Problem-based learning: A strategy to foster generation Z's critical thinking and perseverance. Teaching and Learning in Nursing, 16(1):85-88, 2021.

[16] Liudmila Varenina, Elena Vecherinina, Elena Shchedrina, Ildar Valiev, and Artem Islamov. Developing critical thinking skills in a digital educational environment. Thinking Skills and Creativity, 41:100906, 2021.

[17] Tiffany A Reese, Tamia A Harris-Tryon, Jennifer G Gill, and Laura A Banaszynski. Supporting women in academia during and after a global pandemic, 2021.

[18] Beatrice Avolio, Jessica Chávez, and Carlos Vílchez-Román. Factors that contribute to the underrepresentation of women in science careers worldwide: a literature review. Social Psychology of Education, 23(3):773-794, 2020. 
[19] Naomi Ellemers, Henriette Van den Heuvel, Dick De Gilder, Anne Maass, and Alessandra Bonvini. The underrepresentation of women in science: Differential commitment or the queen bee syndrome? British Journal of Social Psychology, 43(3):315-338, 2004. 